\title{
REFUERZOS DE LOSAS MACIZAS DE EDIFICACIÓN EN HORMIGÓN ARMADO
}

\author{
(SOLID SLAB STRENGTHENING IN REINFORCED CONCRETE)
}

José L. Ramírez, Dr. Ing. Industrial.

José M. Bárcena, Dr. Ing. Industrial.

Pedro J. Landa, Ing. Industrial.

Fecha de recepcion: 3-V.90

LABEIN, Bilbao. ESPAÑA

$454-2$

\section{RESUMEN}

Es frecuente encontrar en edificación forjados a base de losa maciza de hormigón armado que precisan refuerzo, sea por cam. bio de uso o por deterioro, en este caso fundamentalmente debido a la corrosión de armaduras.

El refuerzo de este tipo de elementos estructurales es más viable que el de los constituidos por viguetas debido a su mejor resistencia a todos los efectos derivados del esfuerzo cortante. Con todo, ha parecido interesante a los autores el desarrollar un programa de investigación que evaluara el comportamiento experimental de diversos métodos de refuerzo, desde los más simples e inmediatos a otros con mayor grado de intervención, considerando a la vez la utilización de morteros especiales adhesivos.

Los resultados de los ensayos de rotura de forjados de tamaño próximo al real, reforzados por cinco procedimientos diferentes, y la comparación con su cálculo teórico y con los ensayos de forjados sin reforzar, permiten extraer conclusiones de interés. En algunos casos el comportamiento es muy pueno, en otros existe alguna susceptibilidad a corregir con el coeficiente de seguridad, y en alguno se debe limitar la tasa de incremento de carga por el riesgo de despegue total del refuerzo.

\section{SYNOPSIS}

It is frequent to find buildings made of solid slab reinforced concrete that require strengthening, either because of a change in use or due to deterioration. In the latter case, this is fundamentally caused by corrosion of the reinforcement.

Strengthening this type of structural element is more feasible than for those made of beams thanks to their greater resistance against all the effects derived from the shearing load. Therefore, the authors considered interesting the development of a research program that would evaluate the experimental behaviour of different strengthening methods, from the most simple or inmediate methods to others with greater intervention le. vels, also taking into consideration the use of special adhesive mortars.

The results of the bending tests, carried out almost on a full scale, with five different strengthening procedures and the comparison of their theoretical calculation with the reference slab tests allow interesting conclusions to be reached. In some cases be. haviour is very good while others are susceptible to correction using the appropiate safety factor, and in some cases the load increase rate should be limited due to the strengthening becoming completely detached.

\section{INTRODUCCIÓN}

En el campo de la reparación y refuerzo de estructuras de edificación en hormigón armado, ocupa un lugar propio la problemática concerniente a los forjados de losa maciza con armadura normalmente unidireccional.

En efecto, la mayor parte de los edificios de plantas construidas hasta la década de los cincuenta se proyectaban y realizaban con pisos a base de losa maciza apoyada en vigas, presentando generalmente un espesor sorprendentemente reducido, con armaduras de pequeño diámetro próximas a su cara inferior y, muchas veces, con hormigón de escasa calidad.
En la revisión estructural que modernamente se viene haciendo con motivo de la rehabilitación de edificios antiguos hay principalmente dos problemas que suelen originar un proyecto de refuerzo.

Uno de ellos es la mala conservación, puesta de manifiesto fundamentalmente por la corrosión de las armaduras delgadas y próximas a la superficie, con acompañamiento de grietas longitudinales y desprendimientos del hormigón. El otro es la imposible justificación por cálculo de la resistencia y deformabilidad exigidas para las cargas de servicio que un cambio de uso plantea. 
La necesidad comprobada en nuestro entorno de frecuentes intervenciones en este tipo de edificios ha motivado el desarrollo de un programa de investigación, cuyos resultados se exponen a continuación, orientado a verificar el comportamiento de diversos métodos de refuerzo a flexión, bien por suplemento de la armadura inferior, aumento del espesor de la losa o combinación de ambas actuaciones.

\section{BASES DE CÁLCULO}

Tratándose de forjados unidireccionales a base de losa maciza de hormigón, el planteamiento del cálculo es muy simple, pues pueden utilizarse las fórmulas correspondientes a secciones rectangulares, considerando una banda de ancho determinado.

Debido a la sección maciza de hormigón no existirá, en general, problema de resistencia al esfuerzo cortante. Por ello los refuerzos proyectados están pensados como refuerzo a momento flector.

Aunque, a priori, no aparezca como problemático, convendrá comprobar los esfuerzos rasantes en las caras de contacto de la capa de hormigón de refuerzo, sea ésta inferior o superior, con el forjado original y, en caso necesario, añadir los elementos de cosido correspondientes.

Queda fuera del presente trabajo la consideración de los fenómenos de fluencia y retracción diferenciales, que podrían suponer tensiones normales y rasantes adicionales a tener en cuenta en la práctica, bien comprobando que junto con las de servicio resultan admisibles, sea minimizándolas por un curado adecuado o bien absorbiéndola mediante un enclavamiento entre el forjado y las capas añadidas.

\section{DIMENSIONAMIENTO DE FORJADOS $Y$ REFUERZOS}

En diversos programas parciales se han ido estudiando los tipos de refuerzo de losas macizas que enumeramos a continuación:

- Adhesión de armaduras a la parte inferior de la losa con un mortero adherente ("Armadura inferior gruesan).

- Adhesión de malla electrosoldada a la parte inferior de la losa con un mortero adherente variando respecto al anterior en que las armaduras han sido de pequeño diámetro y menor cuantía ("Armadura inferior reducidan).

- Recrecido por la parte superior con aligeramientos de poliestireno expandido y utilizando conectores metálicos entre los hormigones antiguo y nuevo ("Recrecido superior aligerado y conectores").

- Recrecido por la parte superior sin especial preparación entre la superficie del forjado y la capa añadida ("Recrecido superior»).

- Combinación de recrecido superior sin preparación especial y adhesión de malla electrosoldada inferior con mortero especial adherente ("Combinado superior inferior").

Las losas iniciales de partida se han confeccionado de un espesor de $13 \mathrm{~cm}$, con armadura de acero liso ordinario y hormigones de los niveles inferiores de resistencia, al objeto de asemejarse al tipo de construcción real cuyo refuerzo se ha tratado de evaluar.

Las piezas de ensayo tenían una anchura de $2 \mathrm{~m}$ y $4 \mathrm{~m}$ de distancia entre apoyos.

El análisis de los forjados iniciales de referencia y de los reforzados por los diferentes métodos, se ha efectuado por el método de los estados límites y diagrama parábola-rectángulo. Se han utilizado las características de los materiales en el momento del ensayo y un coeficiente de cansancio del hormigón de 0,95, al efectuarse los ensayos en un tiempo breve. Los planos superpuestos de armaduras se han considerado concentrados en su centro de gravedad con cuantía mecánica equivalente. A continuación se indican los resultados significativos de los análisis realizados.

\subsection{Losas de referencia iniciales}

A lo largo del trabajo ha habido varias etapas fundamentales que, al efectuarlas con cierto intervalo de tiempo han exigido tres losas iniciales de referencia algo diferentes, cuyas características se exponen a continuación.

\subsubsection{Losa de referencia (Fig. 1)}

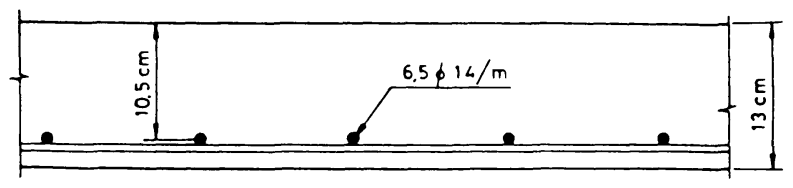

Fig. 1

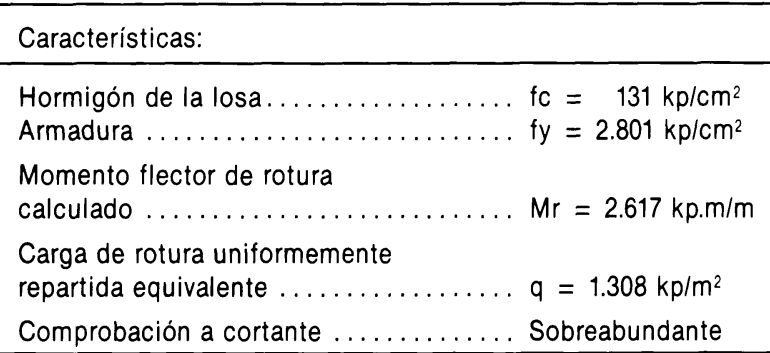




\subsubsection{Losas de referencia 2 y 3 (Fig. 2)}

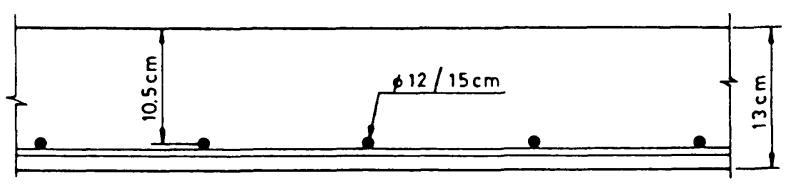

Fig. 2

\begin{tabular}{|c|c|c|}
\hline Caracteristicas: & Losa 2 & Losa 3 \\
\hline $\begin{array}{l}\text { Hormigón de losa... } \\
\text { Armadura ......... }\end{array}$ & $\begin{array}{l}\mathrm{fc}=238 \mathrm{kp} / \mathrm{cm}^{2} \\
\mathrm{fy}=3.554 \mathrm{kp} / \mathrm{cm}^{2}\end{array}$ & $\begin{array}{r}219 \mathrm{kp} / \mathrm{cm}^{2} \\
3.554 \mathrm{kp} / \mathrm{cm}^{2}\end{array}$ \\
\hline $\begin{array}{l}\text { Momento flector de } \\
\text { rotura calculado .... }\end{array}$ & $\mathrm{Mr}=2.650 \mathrm{kp} \cdot \mathrm{m} / \mathrm{m}$ & $2.637 \mathrm{kp} . \mathrm{m} / \mathrm{m}$ \\
\hline $\begin{array}{l}\text { Carga de rotura } \\
\text { uniformemente } \\
\text { repartida }\end{array}$ & & \\
\hline equivalente .... & $\mathrm{q}=1.325 \mathrm{kp} / \mathrm{m}^{2}$ & $1.318 \mathrm{kp} / \mathrm{m}^{2}$ \\
\hline $\begin{array}{l}\text { Comprobación a } \\
\text { cortante ........ }\end{array}$ & \multicolumn{2}{|c|}{ Sobreabundante } \\
\hline
\end{tabular}

\subsection{Losas reforzadas}

\subsubsection{Armadura inferior gruesa (Fig. 3)}

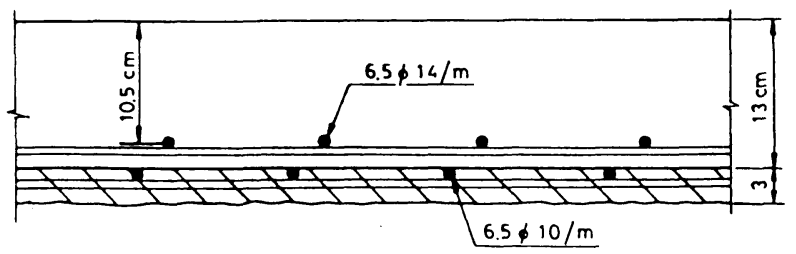

Fig. 3

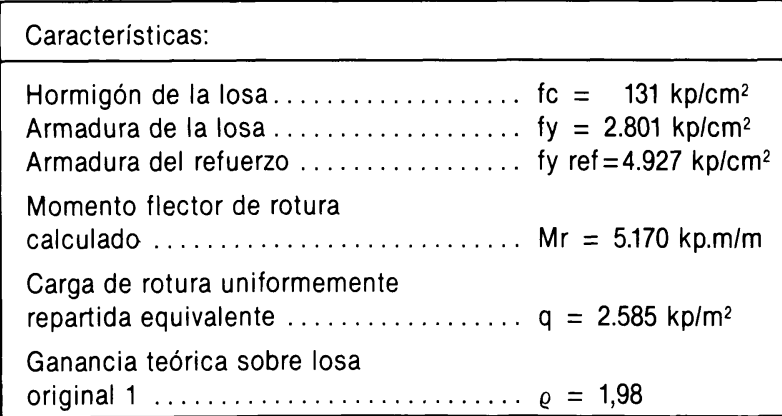

\section{Comprobación a cortante}

Deberemos comprobar la necesidad de armaduras transversales y el comportamiento a rasante en el contacto entre losa original y mortero adherente.

La condición de no necesidad de armaduras transversales se cumple ampliamente.

En cuanto al rasante se puede decir que actúa sobre una superficie de posible rotura frágil, al no existir armadura transversal entre la capa de mortero adheren- te añadida y la losa inicial. En la zona de apoyo, de máximo esfuerzo rasante, se puede suponer, avanzada la carga, estado de fisuración y relación tensiones-deformaciones probablemente no lineal. Estas y algunas otras suposiciones nos llevan a calcular un valor de la solicitación de rasante (3-33.5) en la intercara losarefuerzo, en el momento de la rotura por flexión en nuetras condiciones de ensayo de

$$
\tau \text { máx } \cong 1,9 \mathrm{kp} / \mathrm{cm}^{2}
$$

Los valores resistentes asignados a este tipo de esfuerzo por diversas normas y autores oscilan en un amplio abanico de valores, dependiendo mucho de la rugosidad de las superficies de contacto (2-f 13.21).

\begin{tabular}{|c|c|}
\hline Model Code (superficie rugosa) . . & $3,2 \mathrm{kp} / \mathrm{cm}^{2}$ \\
\hline $\begin{array}{l}\text { ACI-318-83 (superficie intencionadamente } \\
\text { rugosa) } \ldots \ldots \ldots \ldots \ldots \ldots \ldots \ldots \ldots \ldots \ldots\end{array}$ & $6,5 \mathrm{kp} / \mathrm{cm}^{2}$ \\
\hline BAEL-83 (en general) . . & $0 \mathrm{kp} / \mathrm{cm}^{2}$ \\
\hline $\begin{array}{l}\text { CP-110 (superficie cepillada antes de } \\
\text { endurecer) } \ldots \ldots \ldots \ldots \ldots \ldots \ldots \ldots \ldots\end{array}$ & $<3,9 \mathrm{kp} / \mathrm{cm}^{2}$ \\
\hline
\end{tabular}

A la vista de los valores anteriores, en un caso nulo, como en la norma francesa BAEL-83, y en otros superiores al calculado para nuestro forjado en el momento teórico de rotura por flexión, pero con exigencia de superficie intencionadamente rugosa que no es nuestra situación, se puede suponer riesgo de fallo por rasante antes del agotamiento por flexión. Además no hay ningún efecto beneficioso de rozamiento bajo la presión de las cargas, ya que la capa de refuerzo está colgada de la losa inicial.

\subsubsection{Armadura inferior reducida (Fig. 4)}

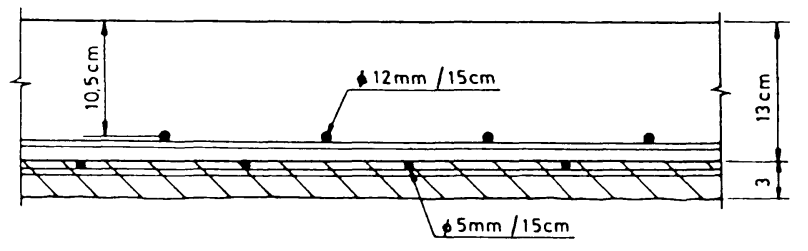

Fig. 4

\begin{tabular}{|c|}
\hline Caracteristicas: \\
\hline $\begin{array}{l}\text { Hormigón de la losa } \ldots \ldots \ldots \ldots \ldots \ldots \text { fc }=238 \mathrm{kp} / \mathrm{cm}^{2} \\
\text { Armadura de la losa } \ldots \ldots \ldots \ldots \ldots \text { fy }=3.554 \mathrm{kp} / \mathrm{cm}^{2} \\
\text { Malla electrosoldada de refuerzo } \ldots \ldots \text { fy } \mathrm{ref}=6.433 \mathrm{kp} / \mathrm{cm}^{2}\end{array}$ \\
\hline $\begin{array}{l}\text { Momento flector de rotura } \\
\text { calculado } \ldots \ldots \ldots \ldots \ldots \ldots \ldots \ldots . . M r=3.697 \mathrm{kp} . \mathrm{m} / \mathrm{m}\end{array}$ \\
\hline $\begin{array}{l}\text { Carga de rotura uniformemente } \\
\text { repartida equivalente ........... }\end{array}$ \\
\hline $\begin{array}{l}\text { Ganancia teórica sobre losa } \\
\text { original } 2 \ldots \ldots \ldots \ldots \ldots\end{array}$ \\
\hline
\end{tabular}




\section{Comprobación a cortante}

Con análogos argumentos y los mismos razonamientos del caso anterior "Armadura inferior gruesa" se concluye lo siguiente:

La condición de no necesidad de armaduras transversales se cumple con amplitud.

La solicitación de rasante en la interfase losa-refuerzo en el momento de rotura por flexión resulta de

$$
\tau \text { máx } \cong 0,6 \mathrm{kp} / \mathrm{cm}^{2}
$$

Recordando las consideraciones de resistencia al esfuerzo rasante hechas en el tipo de refuerzo anterior, pero teniendo en cuenta el valor teórico suficientemente pequeño obtenido, suponemos que, con bastante probabilidad, el fallo se producirá por flexión antes que por rasante.

\subsubsection{Recrecido superior aligerado y conectores} (Fig. 5)
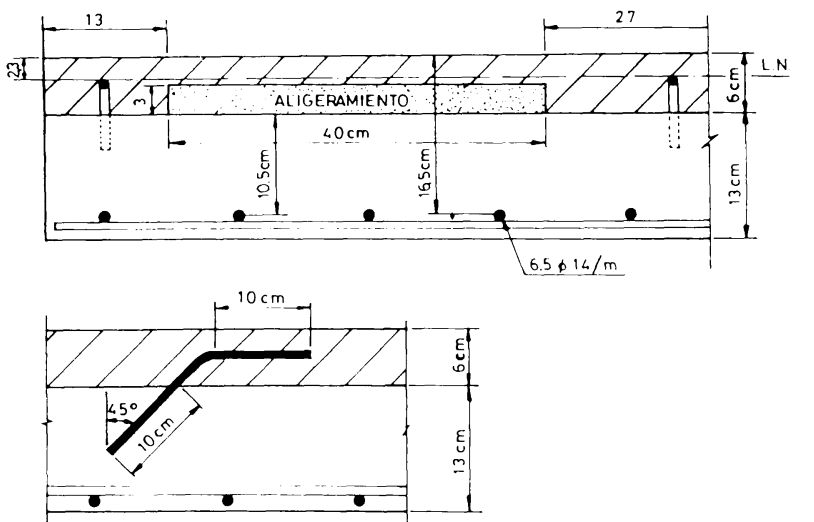

Fig. 5

\begin{tabular}{|c|}
\hline Caracteristicas: \\
\hline 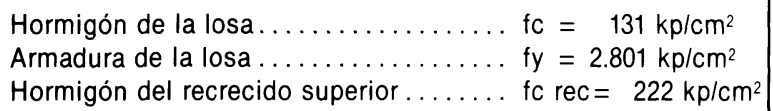 \\
\hline $\begin{array}{l}\text { Momento flector de rotura } \\
\text { calculado } \ldots \ldots \ldots \ldots \ldots \ldots \ldots \ldots . . . \ldots r=4.391 \mathrm{kp} . \mathrm{m} / \mathrm{m}\end{array}$ \\
\hline $\begin{array}{l}\text { Carga de rotura uniformemente } \\
\text { repartida equivalente } \ldots \ldots \ldots \ldots \ldots \ldots q=2.195 \mathrm{kp} / \mathrm{m}^{2}\end{array}$ \\
\hline $\begin{array}{l}\text { Ganancia teórica sobre losa } \\
\text { original } 1 \ldots \ldots \ldots \ldots \ldots .\end{array}$ \\
\hline
\end{tabular}

\section{Comprobación a cortante}

Como en los casos anteriores se comprueba la no necesidad de armadura transversal.

Respecto al rasante en la superficie de contacto losarecrecido, superficie que en este caso está reducida en gran manera por la introducción de aligeramientos en el recrecido, el cálculo con rotura por deslizamiento del tipo frágil, en el supuesto de ausencia de conectores, conduce a una $\tau$ máx. $=5,6 \mathrm{kp} / \mathrm{cm}^{2}$ para el momento de rotura calculado, valor superior al admitido por las Normas antes citadas.

Por ello se ha diseñado este tipo de reparación con la inserción de conectores (Figura 5) que permiten resistir un rasante en la superficie de contacto de $\tau_{u}=8,1 \mathrm{kp} / \mathrm{cm}^{2}$, valor calculado según la fórmula aditiva que tiene en cuenta la adhesión entre hormigones en superficie no especialmente rugosa y las dos componentes generadas por los conectores a $45^{\circ}$ : componente horizontal y rozamiento provocado por la componente vertical (2-13.19).

En este supuesto de recrecidos aligerados no es probable que se pueda absorber el rasante por el simple contacto entre hormigones, pero que si se puede resistir con un adecuado diseño de conectores de forma que sea crítica la flexión antes que el rasante.

\subsubsection{Recrecido superior (Fig. 6)}

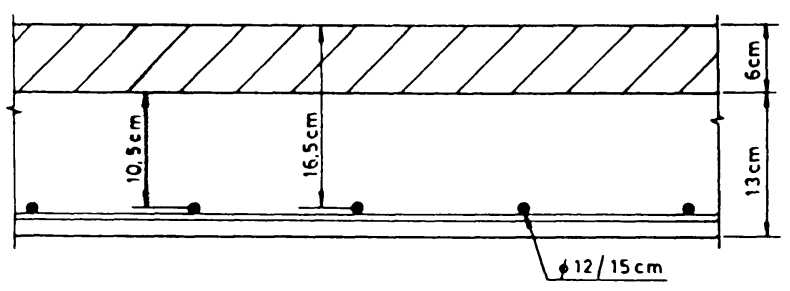

Fig. 6

\begin{tabular}{|c|}
\hline Características: \\
\hline 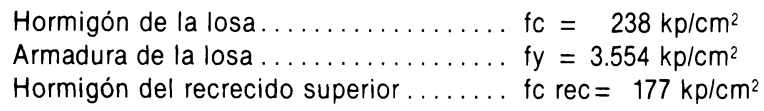 \\
\hline 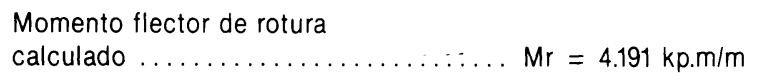 \\
\hline $\begin{array}{l}\text { Carga de rotura uniformemente } \\
\text { repartida equivalente ........... }\end{array}$ \\
\hline $\begin{array}{l}\text { Ganancia teórica sobre losa } \\
\text { original } 2 \ldots \ldots \ldots \ldots \ldots \ldots \ldots \ldots\end{array}$ \\
\hline
\end{tabular}

\section{Comprobación a cortante}

No hay necesidad de armadura transversal.

El rasante máximo en la zona de contacto entre los dos hormigones, en el supuesto de rotura frágil por ausencia de armaduras, vale $\tau$ máx. $=2,2 \mathrm{kp} / \mathrm{cm}^{2}$ para el momento flector de rotura de la losa reforzada.

El cálculo de $\tau_{u}$ para contacto entre superficies no especialmente rugosas resulta en $\tau_{u}=4,2 \mathrm{kp} / \mathrm{cm}^{2}$.

Habida cuenta de la diferencia entre 2,2 y $4,2 \mathrm{kp} / \mathrm{cm}^{2}$, y que la capa añadida está en la parte superior con 
lo que tanto su peso propio como las cargas actúan favoreciendo un rozamiento entre capas, hay que pensar que será crítica la flexión antes que el rasante.

\subsubsection{Combinado superior inferior (Fig. 7)}

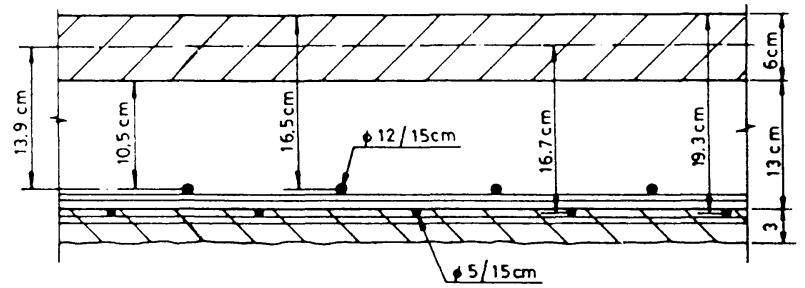

Fig. 7

\begin{tabular}{|c|}
\hline Caracteristicas: \\
\hline $\begin{array}{l}\text { Hormigón de la losa } \ldots \ldots \ldots \ldots \ldots \ldots \ldots \text { fc }=219 \mathrm{kp} / \mathrm{cm}^{2} \\
\text { Hormigón recrecido superior } \ldots \ldots \ldots \ldots \text { fc } \mathrm{rec}=233 \mathrm{kp} / \mathrm{cm}^{2} \\
\text { Armadura de la losa } \ldots \ldots \ldots \ldots \ldots \ldots \text { fy }=3.554 \mathrm{kp} / \mathrm{cm}^{2} \\
\text { Malla electrosoldada de refuerzo } \ldots \ldots \ldots \text { fy } \text { ref }=6.433 \mathrm{kp} / \mathrm{cm}^{2}\end{array}$ \\
\hline $\begin{array}{l}\text { Momento flector de rotura } \\
\text { calculado } \ldots \ldots \ldots \ldots \ldots \ldots \ldots \ldots \text { Mr }=5.805 \mathrm{kp} . \mathrm{m} / \mathrm{m}\end{array}$ \\
\hline $\begin{array}{l}\text { Carga de rotura uniformemente } \\
\text { repartida equivalente } \ldots \ldots \ldots \ldots \ldots \ldots q=2.902 \mathrm{kp} / \mathrm{m}^{2}\end{array}$ \\
\hline $\begin{array}{l}\text { Ganancia teórica sobre losa } \\
\text { original } 3 \ldots \ldots \ldots \ldots \ldots \ldots \ldots \ldots \ldots \ldots\end{array}$ \\
\hline
\end{tabular}

\section{Comprobación a cortante}

Como en todos los casos no hay necesidad de armadura transversal.

Respecto al rasante existen aqui dos superficies de comprobación.

En el contacto entre losa original y recrecido superior se producirá, para el nuevo momento flector, en el supuesto de rotura frágil por ausencia de armaduras, un valor $\tau$ máx. $=2,9 \mathrm{kp} / \mathrm{cm}^{2}$ inferior al $\tau_{u}=4,7 \mathrm{kp} / \mathrm{cm}^{2}$ de agotamiento para superficies no especialmente rugosas. El actuar las cargas comprimiendo la superficie de contacto dará una componente de rozamiento que favorecerá el buen comportamiento a rasante.

En la cara inferior de contacto del mortero adherente con la losa original resulta un valor de $\tau$ máx. $=0,6 \mathrm{kp} / \mathrm{cm}^{2}$, que por su valor tan reducido es muy probable sea resistido hasta la rotura por flexión de la losa reforzada, a pesar de que a ese tipo de intercara sea muy dudosa la atribución de un valor sig. nificativo de resistencia a rasante.

\section{EJECUCIÓN DE LOS REFUERZOS}

En las figuras 1 a 7 se han mostrado las secciones transversales de las losas tanto originales como con (c) Consejo Superior de Investigaciones Científicas Licencia Creative Commons 3.0 España (by-nc) los cinco tipos de refuerzo. En el apartado anterior se han dado las calidades reales de los materiales utilizados.

Las losas a reforzar han sido aligeradas de gran parte de su peso propio, previamente al proceso de refuerzo, gracias a un apuntalamiento activo.

\section{"Armadura inferior gruesa" (Fig. 3)}

No se ha hecho ninguna preparación de la superficie inferior del forjado original, hormigonado sobre encofrado de madera.

Las nuevas armaduras de refuerzo se han colocado en contacto con dicha superficie inferior, sujetas contra ella por medio de pequeños anclajes con tuerca.

Antes de la aplicación del mortero adherente que recubre la armadura se ha aplicado una capa de imprimación con un adhesivo tipo látex. Posteriormente el mortero adherente ha sido aplicado en capas sucesivas con Ilana, hasta lograr un completo recubrimiento de la armadura.

El mortero ha sido del tipo modificado con polímeros, desarrollando unos $250 \mathrm{kp} / \mathrm{cm}^{2}$ de resistencia a la compresión a los 28 dias, para una relación agua/producto de 0,2 . El endurecimiento es rápido presentando unos $100 \mathrm{kp} / \mathrm{cm}^{2}$ a las 24 horas.

Este método tiene el inconveniente del coste alto del material y del elevado trabajo que exige su colocación, pues es necesario aplicarlo en pequeñas capas para evitar que se descuelgue, habiéndose de esperar varias horas entre ellas. Por ello, desde el punto de vista económico no será un método adecuado para grandes superficies, en cuyo caso el hormigón proyectado podría ser más recomendable.

\section{"Armadura inferior reducida" (Fig. 4)}

Idéntico proceso al anterior. La única diferencia es la de utilización de malla electrosoldada de $\varnothing 5 \mathrm{~mm}$, en vez de malla confeccionada por ferrallistas con armaduras principales de $\varnothing 10 \mathrm{~mm}$.

\section{"Recrecido superior aligerado y conectores" (Fig. 5)}

Como se deduce de la figura, el hormigón de refuerzo se ha vertido sobre la superficie superior de la losa sin tratamiento especial, en la que previamente se habian anclado con resina poliéster los conectores a $45^{\circ}$, en agujeros perforados con taladro percutor (Foto 1). Se habian también dispuesto tres líneas de poliestireno expandido de $3 \mathrm{~cm}$ de espesor y $40 \mathrm{~cm}$ de anchura cada una (Foto 2).

http://informesdelaconstruccion.revistas.csic.es 


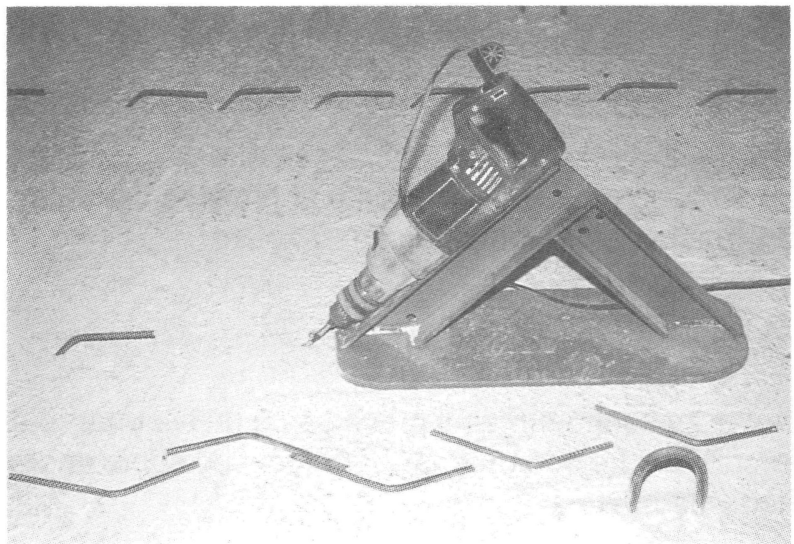

Foto 1

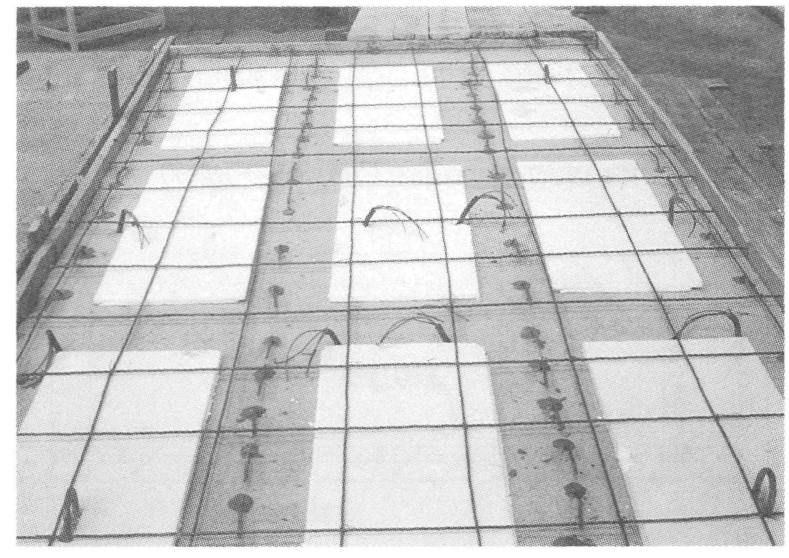

Foto 2

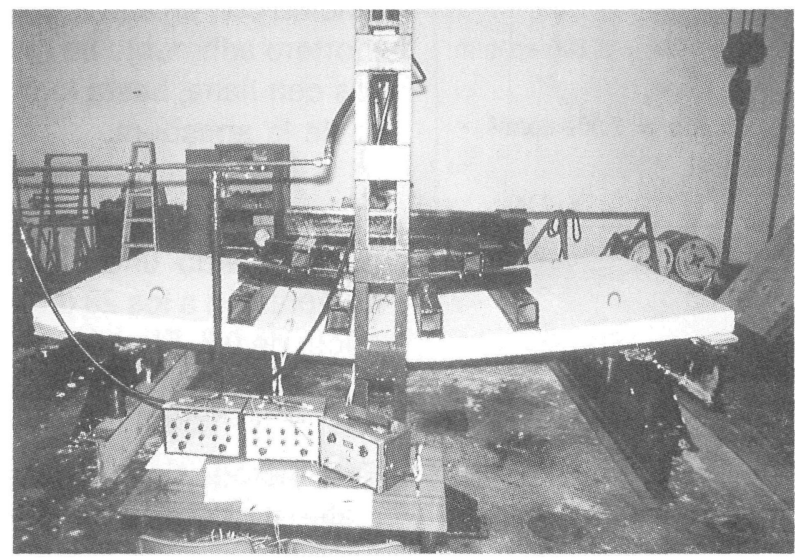

Foto 3

«Recrecido superior» (Fig. 6)

En este caso se ha vertido simplemente la capa de refuerzo de $6 \mathrm{~cm}$ de espesor sobre la superficie superior de la losa original, sin preparación previa.

\section{"Combinado superior inferior» (Fig. 7)}

Se trata de la realización conjunta del $2 .^{\circ}$ y $4 .^{\circ}$ métodos descritos.

\section{DESARROLLO DE LOS ENSAYOS}

Las diferentes losas, originales y reforzadas, de una dimensión de $2 \times 4 \mathrm{~m}$ se han ensayado, simplemente apoyadas, a flexión en la dirección longitudinal. El programa descrito se ha basado en 20 losas de ensayo.
Las cargas se aplicaron en cuatro líneas transversales separadas $1 / 7$ de la luz entre sí, quedando las líneas exteriores a 2/7 de la luz de los apoyos respectivos (Foto 3).

El proceso de carga ha consistido en una subida sucesiva por escalones hasta la carga de servicio, descenso hasta nivel de peso propio, y nueva subida posterior escalonada hasta la rotura. Durante la misma se han medido y registrado por medio de transductores y extensómetros óhmicos flechas y tensiones en las armaduras, además de anotarse el proceso de agrietamiento y otras incidencias.

En el Cuadro 1 se exponen los resultados más importantes obtenidos, tanto a nivel individual de las 20 losas ensayadas, como los valores medios por grupos. 


\section{CUADRO 1 \\ RESULTADOS ENSAYO FLEXIÓN LOSAS}

\begin{tabular}{|c|c|c|c|c|c|c|}
\hline \multirow{2}{*}{$\begin{array}{l}\text { Tipo de losa } \\
\text { y refuerzo }\end{array}$} & \multirow{2}{*}{$N .^{0}$} & \multicolumn{2}{|c|}{$\begin{array}{l}\text { Momento de } \\
\text { rotura } \\
\mathrm{kp} . \mathrm{m} / \mathrm{m}\end{array}$} & \multicolumn{2}{|c|}{$\begin{array}{l}\text { Flecha al } 35 \% \\
\text { carga de rotura } \\
\left({ }^{\star}\right)(\mathrm{mm})\end{array}$} & \multirow{2}{*}{$\begin{array}{l}\text { Tipo de } \\
\text { rotura }\end{array}$} \\
\hline & & Ind. & Med. & Ind. & Med. & \\
\hline $\begin{array}{l}\text { Losa } \\
\text { de } \\
\text { referencia } 1 .\end{array}$ & $\begin{array}{l}1 \\
2 \\
3\end{array}$ & $\begin{array}{l}3114 \\
3150 \\
3257\end{array}$ & 3174 & $\begin{array}{l}1,8 \\
2,6 \\
3,8\end{array}$ & 2,7 & $\begin{array}{l}\text { Agotamiento a flexión } \\
\text { con rotura de } \\
\text { armaduras }\end{array}$ \\
\hline $\begin{array}{l}\text { Losa } \\
\text { de } \\
\text { referencia } 2 .\end{array}$ & $\begin{array}{l}1 \\
2\end{array}$ & $\begin{array}{l}3516 \\
3328\end{array}$ & 3422 & $\begin{array}{l}3,3 \\
4,3\end{array}$ & 3,8 & $\begin{array}{l}\text { Agotamiento a flexión } \\
\text { con rotura de } \\
\text { armaduras }\end{array}$ \\
\hline $\begin{array}{l}\text { Losa } \\
\text { de } \\
\text { referencia } 3 .\end{array}$ & $\begin{array}{l}1 \\
2\end{array}$ & $\begin{array}{l}3255 \\
3305\end{array}$ & 3280 & $\begin{array}{l}3,1 \\
2,5\end{array}$ & 2,8 & $\begin{array}{l}\text { Agotamiento a flexión } \\
\text { con rotura de } \\
\text { armaduras }\end{array}$ \\
\hline $\begin{array}{l}\text { Armadura } \\
\text { inferior } \\
\text { gruesa }\end{array}$ & $\begin{array}{l}1 \\
2 \\
3\end{array}$ & $\begin{array}{l}4279 \\
6127 \\
3570\end{array}$ & 4659 & $\begin{array}{l}3,9 \\
6,1 \\
1,4\end{array}$ & 3,8 & $\begin{array}{l}\text { Deslizamiento refuerzo } \\
\text { Flexión y deslizamiento } \\
\text { Despegue refuerzo }\end{array}$ \\
\hline $\begin{array}{l}\text { Armadura } \\
\text { inferior } \\
\text { reducida }\end{array}$ & $\begin{array}{l}1 \\
2 \\
3\end{array}$ & $\begin{array}{l}4290 \\
4290 \\
4255\end{array}$ & 4278 & $\begin{array}{l}3,2 \\
2,0 \\
2,1\end{array}$ & 2,4 & $\begin{array}{l}\text { Agotamiento a flexión } \\
\text { con rotura de armadu- } \\
\text { ras del refuerzo }\end{array}$ \\
\hline $\begin{array}{l}\text { Recrecido superior } \\
\text { aligerado y } \\
\text { conectores }\end{array}$ & $\begin{array}{l}1 \\
2 \\
3\end{array}$ & $\begin{array}{l}5428 \\
5535 \\
5635\end{array}$ & 5533 & $\begin{array}{l}1,9 \\
1,9 \\
2,3\end{array}$ & 2,0 & $\begin{array}{l}\text { Agotamiento a flexión } \\
\text { con rotura de } \\
\text { armaduras }\end{array}$ \\
\hline $\begin{array}{l}\text { Recrecido } \\
\text { superior }\end{array}$ & $\begin{array}{l}1 \\
2\end{array}$ & $\begin{array}{l}4752 \\
4602\end{array}$ & 4677 & $\begin{array}{l}1,8 \\
1,2\end{array}$ & 1,5 & $\begin{array}{l}\text { Rotura de armaduras e } \\
\text { inicio de despegue de } \\
\text { la capa superior }\end{array}$ \\
\hline $\begin{array}{l}\text { Combinado } \\
\text { superior } \\
\text { e inferior }\end{array}$ & $\begin{array}{l}1 \\
2\end{array}$ & $\begin{array}{l}6393 \\
6393\end{array}$ & 6393 & $\begin{array}{l}1,1 \\
1,6\end{array}$ & 1,4 & $\begin{array}{l}\text { Agotamiento a flexión } \\
\text { con rotura de armadu- } \\
\text { ras del refuerzo }\end{array}$ \\
\hline
\end{tabular}

(*) Se considera que el $35 \%$ de la carga de rotura en un ensayo rápido vale aproximadamente el valor de la carga de servicio de la losa. La flecha correspon. de a la acción de las sobrecargas.

\section{MODOS DE ROTURA}

De forma genérica todas las losas de referencia han roto por flexión, con rotura de armaduras, acompañada de una gran deformación correspondiente a la elevada flexibilidad de las mismas.

Análogo tipo de rotura han presentado las losas con recrecido superior aligerado más conectores y recrecido superior simple, salvo que en este último caso, al no existir conectores, se producia en la proximidad de la rotura un comienzo de despegue del recrecido, al no ser arrastrado por ningún anclaje en la fuerte incurvación del forjado.
En las losas con refuerzo inferior se han producido diferentes formas de rotura, desde la de las armaduras añadidas hasta el desprendimiento total del refuerzo de una de las losas, dependiendo de la cuantía mecánica de las armaduras suplementarias.

Las piezas con armaduras de refuerzo de diametro «pequeño" presentan una homogeneidad de resultados, tanto en cuanto a carga de rotura como a la forma de hacerlo: rotura de las armaduras de refuerzo. En cambio las piezas con armaduras de refuerzo "gruesas" tienen un comportarniento totalmente irregular aunque se presenta una clara tendencia al deslizamiento y despegue de la capa de refuerzo (en un caso hay un despegue total) (Foto 4). 


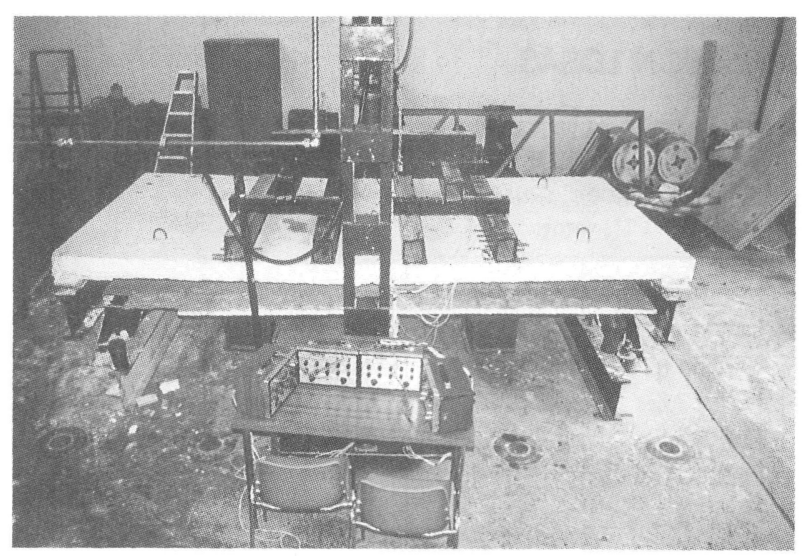

Foto 4

Las losas con refuerzo inferior y superior presentan una rotura combinación de las anteriores, decantándose de forma clara hacia el caso de rotura por flexión al romper armaduras del refuerzo, pero presentándose también un comienzo de despegue del recrecido superior.

\section{RELACIÓN VALORES ENSAYO/CÁLCULO}

En los Cuadros 2 y 3 aparecen reflejados los valores de las relaciones $\gamma_{n}$ entre las cargas de rotura a flexión obtenidas de los ensayos (Cuadro 1) y los calculados para cada tipo de losa original y refuerzo en el apartado 3. Para las losas reforzadas se dan igualmente los valores relativos de su $\gamma_{n}$ medio respecto a los de la losa original.

\section{CONCLUSIONES}

Las observaciones recogidas en los ensayos, en particular su forma de rotura y el análisis de los cuadros de valores, permite deducir una serie de conclusiones. Con referencia a estos cuadros es interesante considerar los valores $\gamma_{\mathrm{n}}$, relación de momentos de rotura real y calculado, que deben ser mayor que la unidad para que el comportamiento real de la losa reparada supere la prestación proyectada para la reparación, así como también los $\gamma_{n}$ relativos respecto a los de la losa de referencia, que orienta sobre las diferencias de comportamiento de losa reforzada y original.

- De los cinco métodos de reparación descritos, cuatro de ellos han logrado $\bar{\gamma}_{n}>1(1.16,1.26,1.12,1.10)$. En cambio, el denominado "Armadura inferior gruesa" ha conducido a un $\bar{\gamma}_{n}=0,9$, lo que indica un comportamiento real por debajo del calculado. Pero el mayor problema con este tipo de refuerzo ha sido la gran dispersión de resultados y la brusquedad de la rotura. En un caso, como se reflejó en el apartado 6, se desprendió repentinamente la armadura inferior para una carga del orden del $60 \%$ de la obtenida en otro ensayo.

Los resultados confirman para el sistema "Armadura inferior gruesa" las hipótesis que se hacian en el apartado 3 sobre el riesgo de fallo por rasante antes del agotamiento por flexión. Además, el mayor diámetro de las armaduras habrá generado una fuerza de despegue normal a la superficie de

CUADRO 2

MÁRGENES DE SEGURIDAD LOSAS DE REFERENCIA

\begin{tabular}{|c|c|c|c|c|}
\hline \multirow{2}{*}{$\begin{array}{l}\text { Losa de } \\
\text { referencia }\end{array}$} & \multicolumn{2}{|c|}{$\begin{array}{l}\text { Momento de rotura } \\
(\mathrm{kp} . \mathrm{m} / \mathrm{m})\end{array}$} & \multirow{2}{*}{$\begin{array}{l}\text { Modo de } \\
\text { rotura }\end{array}$} & \multirow{2}{*}{$\begin{array}{l}\gamma_{n} \\
(2) \\
(1)\end{array}$} \\
\hline & $\begin{array}{l}\text { Cálculo } \\
\text { (1) }\end{array}$ & $\begin{array}{l}\text { Real } \\
\text { (2) }\end{array}$ & & \\
\hline \multirow[t]{2}{*}{1} & \multirow[t]{2}{*}{2617} & $\begin{array}{l}3114 \\
3150 \\
3257\end{array}$ & \multirow{2}{*}{$\begin{array}{l}\text { Agotamiento } \\
\text { a flexión } \\
\text { con rotura de } \\
\text { armaduras }\end{array}$} & $\begin{array}{l}1,19 \\
1,20 \\
1,24\end{array}$ \\
\hline & & $\bar{X} 3174$ & & $\bar{\gamma}_{n} 1,21$ \\
\hline \multirow[t]{2}{*}{2} & \multirow[t]{2}{*}{2650} & $\begin{array}{l}3516 \\
3328\end{array}$ & \multirow[t]{2}{*}{ Idem } & $\begin{array}{l}1,33 \\
1,25\end{array}$ \\
\hline & & $\times 3422$ & & $\bar{\gamma}_{n} 1,29$ \\
\hline \multirow[t]{2}{*}{3} & \multirow[t]{2}{*}{2637} & $\begin{array}{l}3255 \\
3305\end{array}$ & \multirow[t]{2}{*}{ Idem } & $\begin{array}{l}1,23 \\
1,25\end{array}$ \\
\hline & & $\bar{x} 3280$ & & $\bar{\gamma}_{n} 1,24$ \\
\hline
\end{tabular}


CUADRO 3

MÁRGENES DE SEGURIDAD LOSAS REFORZADAS

\begin{tabular}{|c|c|c|c|c|c|}
\hline \multirow{2}{*}{$\begin{array}{l}\text { Tipo } \\
\text { de } \\
\text { losa } \\
\text { reforzada }\end{array}$} & \multicolumn{2}{|c|}{$\begin{array}{l}\text { Momento de rotura } \\
(\mathrm{kp} . \mathrm{m} / \mathrm{m})\end{array}$} & \multirow{2}{*}{$\begin{array}{l}\text { Modo de } \\
\text { rotura }\end{array}$} & \multirow{2}{*}{$\begin{array}{l}\gamma_{\mathrm{n}} \\
\frac{(2)}{(1)}\end{array}$} & \multirow{2}{*}{$\begin{array}{l}\qquad \bar{\gamma}_{n} \\
\text { relativo a } \\
\text { la losa de } \\
\text { referencia } \\
\text { (i) }\end{array}$} \\
\hline & $\begin{array}{c}\text { Cálculo } \\
\text { (1) }\end{array}$ & $\begin{array}{l}\text { Real } \\
(2)\end{array}$ & & & \\
\hline \multirow{2}{*}{$\begin{array}{l}\text { Armadura } \\
\text { inferior } \\
\text { gruesa }\end{array}$} & \multirow[t]{2}{*}{5170} & $\begin{array}{l}4279 \\
6127 \\
3570\end{array}$ & \multirow[t]{2}{*}{$\begin{array}{l}\text { Desliz. refuerzo } \\
\text { Flexión y deslizamiento } \\
\text { Despegue y refuerzo }\end{array}$} & $\begin{array}{l}0,83 \\
1,18 \\
0,69\end{array}$ & (1) \\
\hline & & $\bar{X} 4659$ & & $\bar{\gamma}_{n} 0,90$ & 0,74 \\
\hline \multirow{2}{*}{$\begin{array}{l}\text { Armadura } \\
\text { inferior } \\
\text { reducida }\end{array}$} & \multirow[t]{2}{*}{3697} & $\begin{array}{l}4290 \\
4290 \\
4255\end{array}$ & \multirow{2}{*}{$\begin{array}{l}\text { Agotamiento a } \\
\text { flexión con } \\
\text { rotura de } \\
\text { armaduras del } \\
\text { refuerzo }\end{array}$} & $\begin{array}{l}1,16 \\
1,16 \\
1,15\end{array}$ & (2) \\
\hline & & $\bar{X} 4278$ & & $\bar{\gamma}_{\mathrm{n}} 1,16$ & 0,90 \\
\hline \multirow{2}{*}{$\begin{array}{l}\text { Recrecido } \\
\text { superior } \\
\text { aligerado } \\
\text { y conectores }\end{array}$} & \multirow[t]{2}{*}{4391} & $\begin{array}{l}5428 \\
5535 \\
5635\end{array}$ & \multirow[t]{2}{*}{$\begin{array}{l}\text { Agotamiento a } \\
\text { flexión con } \\
\text { rotura de } \\
\text { armaduras }\end{array}$} & $\begin{array}{l}1,24 \\
1,26 \\
1,28\end{array}$ & (1) \\
\hline & & $\bar{X} 5533$ & & $\bar{\gamma}_{\mathrm{n}} 1,26$ & 1,04 \\
\hline \multirow[t]{2}{*}{$\begin{array}{l}\text { Recrecido } \\
\text { superior }\end{array}$} & \multirow[t]{2}{*}{4191} & $\begin{array}{l}4752 \\
4602\end{array}$ & \multirow{2}{*}{$\begin{array}{l}\text { Rotura de armaduras } \\
\text { e inicio de } \\
\text { despegue de } \\
\text { la capa inferior }\end{array}$} & $\begin{array}{l}1,13 \\
1,10\end{array}$ & (2) \\
\hline & & $\bar{X} 4677$ & & $\bar{\gamma}_{n} 1,12$ & 0,87 \\
\hline \multirow{2}{*}{$\begin{array}{l}\text { Combinado } \\
\text { superior } \\
\text { e inferior }\end{array}$} & \multirow[t]{2}{*}{5805} & $\begin{array}{l}6393 \\
6393\end{array}$ & \multirow{2}{*}{$\begin{array}{l}\text { Agotamiento a } \\
\text { flexión con } \\
\text { rotura de } \\
\text { armaduras del } \\
\text { refuerzo }\end{array}$} & $\begin{array}{l}1,10 \\
1,10\end{array}$ & (3) \\
\hline & & $\bar{x} 6393$ & & $\bar{\gamma}_{\mathrm{n}} 1,10$ & 0,89 \\
\hline
\end{tabular}

contacto, a medida que la deformación de la losa ha ido progresando, debido a su importante rigidez a la flexión propia.

La diferencia entre este método y el denominado "Armadura inferior reducida", que ha presentado un buen comportamiento, es el mayor nivel de incremento de resistencia proyectado, $98 \%$ frente a $40 \%$, y el mayor diámetro de las armaduras, $10 \mathrm{~mm}$ frente a $5 \mathrm{~mm}$. Se deduce pues la necesidad de limitar la tasa de refuerzo a valores moderados (por ejemplo $<50 \%$ ) y emplear armadura de pequeño diámetro, con menor rigidez a la flexión.

- De los cuatro métodos de refuerzo con $\bar{\gamma}_{n}>1$, sólo ha habido uno, el denominado "Recrecido superior aligerado y conectores", que ha mejorado incluso su $\gamma_{n}$ respecto al de la losa original $\left(\bar{\gamma}_{n}\right.$ rel $=$ $=1,04)$.
Se trata de un método interesante y seguro de refuerzo que, además, ha permitido incrementos de carga importantes $(\varrho=1,68)$.

- Los métodos "Armadura inferior reducida", "Recrecido superior" y "Combinado superior inferior", con tasas de incremento de resistencia de 40\%, 58\% y $120 \%$ respectivamente, han superado en los ensayos su resistencia de proyecto $\bar{\gamma}_{n}>1(1.16,1.12$, 1.10). Sin embargo se observa en ellos alguna susceptibilidad al efecto de la reparación puesto que, relativamente a sus correspondientes losas originales, los $\gamma_{n}$ son algo menores: $\bar{\gamma}_{n}$ rel $<1(0.90$, $0.87,0.89$ ).

Para proporcionar a estos tipos de refuerzo una seguridad análoga a la de las piezas originales, se desprende que habría que afectar al cálculo de los mismos con un coeficiente de seguridad adicional del orden de 1,1 . 
- En el método "Recrecido superior», añadiendo una capa superior de hormigón sin ninguna conexión especial con la losa original, aunque el comportamiento general ha sido bueno, se ha observado en las proximidades de la rotura un despegue y levantamiento de la capa de refuerzo, atribuible a dicha falta de conexión. En los casos reales, con cargas más repartidas sobre la superficie que en los ensayos en que la carga se ha aplicado sobre cuatro líneas, la situación es más favorable pues las cargas comprimen la capa de refuerzo contra la losa original.

- Finalmente, desde el punto de vista de deformabilidad, los resultados han tenido parecida significación a los de resistencia. A excepción del refuerzo denominado "Armadura inferior gruesa" los otros cuatro tipos han dado flechas para las correspondientes cargas de servicio, estimadas en el $35 \%$ de las de agotamiento en el ensayo, inferiores a las de la losa original de partida, por lo que desde este punto de vista no puede ponerse ninguna reserva a dichos métodos.

\section{BIBLIOGRAFÍA}

1. Instrucción para el proyecto y la ejecución de obras de hormigón en masa o armado EH-88.

2. CALAVERA, J. "Cálculo, construcción y patología de forjados de edificación" 1988

3. CALAVERA, J. "Proyecto y cálculo de estructuras de hormigón armado para edificios" 1985.

4. CEB 162 (1983). Assessment of concrete structures and design procedures for upgrading (redesign). Praga $228 \mathrm{p}$.

5. FIP (1985). Maintenance, repair and strengthening of existing structures.

6. FERNÁNDEZ CÁNOVAS, M. (1984). Patología y terapéutica del hormigón armado. Dossat, S. A., Madrid 620 p.

7. RAMÍREZ, J. L., BÁRCENA, J. M., LANDA, P. J. "Flexural strengthening for centre moment of joist construction slabs". Structural Fault and Repair 89. Int. Conf. London, June 1989

\section{publicación del IETcc / CSIC}

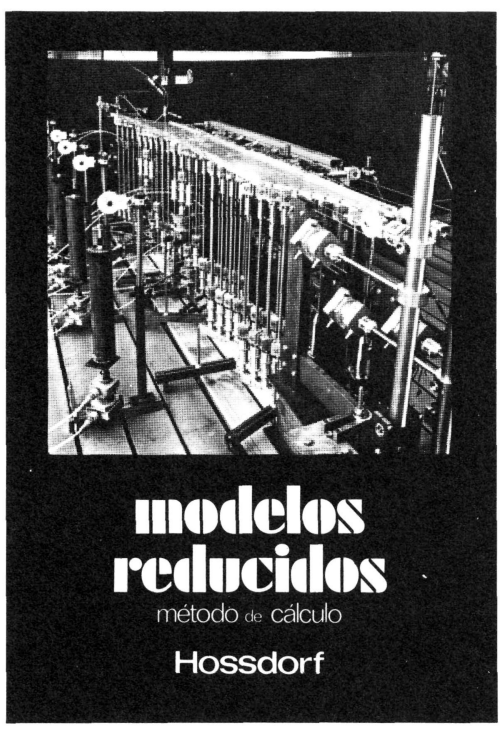

Modelos reducidos. Método de cálculo

H. Hossdorf, Ingeniero Civil

La técnica de los ensayos en modelos reducidos de estructuras sufre hoy dia una decisiva metamorfosis. Hasta hace poco era un medio más bien de artesania, que no siempre era tomado en serio por los academicos teorizantes para comprender el comportamiento resistente de las estructuras complejas y al que se acudió las más de las veces, como a un último remedio debido a sus indiscutibles insuficien cias. Sin embargo, en poco tiempo y gracias a su conexión con los ordenadores digitales, se ha trans formado en un instrumento cientificamente valioso que no puede quedar a un lado en la práctica diaria del Ingeniero Proyectista.

Un volumen encuadernado en cartoné plastificado con lomo de tela, de $17 \times 24 \mathrm{~cm}$, compuesto de 250 páginas, 158 figuras y fotografias.

Precios: 1.800 ptas.; \$ USA 26.00 .

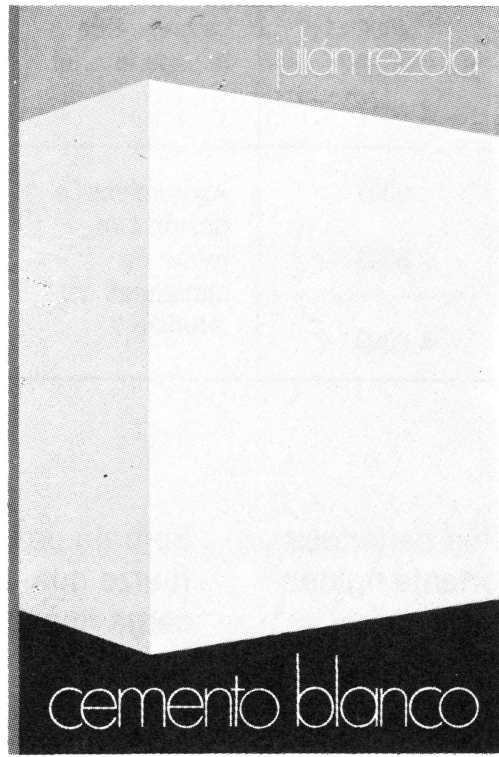

Cemento blanco

Julián Rezola

Ingeniero Químico Dipl. I. Q. S.

Sabido es que existe una extensa y documentada bibliografia sobre el cemento gris: en cambio, no puede decirse lo mismo acerca del cemento portlan blanco, ya que los escritos existentes se refieren tan sólo a algunas peculiaridades que le distinguen de aquél.

El autor nos ofrece sus profundos conocimientos y su larga experiencia tanto en laboratorio como

La parte descriptiva del libro se complementa con gráficos, diagramas y fotografias de gran utilidad, destinados a conseguir la aplicación apropiada de este aglomerante.

Un volumen encuadernado en cartoné policerado, de $17,4 \times 24,3 \mathrm{~cm}$, compuesto de 395 páginas, numerosas fiquras, tablas $\mathrm{v}$ àbacn

Precios: España, 1.700 ptas.; extranjero, \$ 24

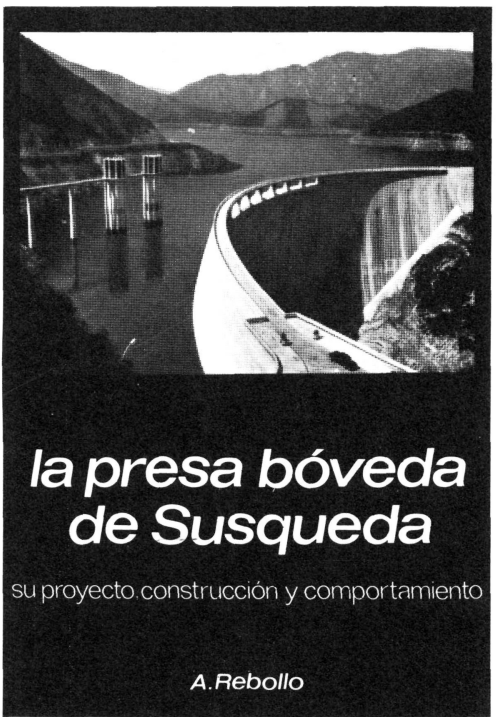

La presa bóveda de Susqueda

A. Rebollo,

Dr. Ingeniero de Caminos

El esfuerzo del constructor de presas se sitúa por su pretensión de perennidad, a contracorriente rizada por lo fung de lavilización actual, caractegrandes presas en funcionamiento 0 en las 10.000 que están envejeciendo $y$ reclaman los cuidados gerontológes para mantener $y$ perfeccionar su servicio y garantizar su inalienable pretensión su perennidad En la medida en que todas nuevas obras, grandes o pequeñas, son portadoras de riesgos ecológicos $y$ a veces, catastróficos, de aumentan con el envejecimiento, la gerontos, que las presas es todo un emplazo. La accion alogia de de Arturo Rebollo en este terreno marca un camino a seguir para todos los que aman su propia camino la devoción paternal que él ha puesto en Susqueda.

Un volumen encuadernado en cartoné plastificado con lomo de tela, de $18 \times 24.5 \mathrm{~cm}$, compuesto de 408 páginas, 330 figuras y fotografias y 39 tablas.

Precios: 1.700 ptas.; extranjero, \$ USA 24.00. 